\title{
Regularities of Evolution from Biopolymers to Social Systems
}

\author{
VA Dementiev* \\ Institute of geochemistry and analytical chemistry RAS, Moskov, Russia \\ *Corresponding author: VA Dementiev, V Vernadsky Institute of geochemistry and analytical chemistry RAS, Moskov, \\ Russia
}

\begin{tabular}{|c|c|}
\hline ARTICLE INFO & ABSTRACT \\
\hline Received: 慧 November 16, 2020 & Citation: VA Dementiev. Regularities of Evolution from Biopolymers to Social Systems. \\
\hline Published: November 24, 2020 & Biomed J Sci \& Tech Res 32(1)-2020. BJSTR. MS.ID.005209. \\
\hline
\end{tabular}

\section{Introduction}

This review is devoted to the author's series of works performed in 2003-2020 within the framework of the Program of the Presidium of the Russian Academy of Sciences "Evolution of the Biosphere". The program was curated by academician E.M. Galimov, who formulated the concept of evolution for the world of polypeptides and polynucleotides in the book The Phenomenon of Life [1]. The tasks of the author were as follows.

a) Addition of physical mechanisms to the collection of biochemical data involved by E.M. Galimov, on the basis of which he constructed a hypothetical scenario of the spontaneous emergence of the genetic code and stable reproduction of protein texts accidentally found by Nature.

b) To logically prove the efficiency of the mentioned scenario using molecular modeling methods.

c) To prove Galimov's hypothesis that in the ancient world of amino acids, nitrogenous bases and ATP, a primitive genetic code could arise, where four nitrogenous bases encoded polypeptides consisting of sequences of only four amino acid residues.

d) To clarify the physical conditions under which amino acids, nitrogenous bases and ATP, dissolved in a neutral medium, could spontaneously create a relatively complex world of polypeptides and nucleotides reproduced in history.

e) Based on historical data, to confirm the validity of Galimov's assumption about the universality of the laws of evolution in any natural systems, up to social ones.
As a result of the accomplishment of these tasks, the author was able to concretize the Galimov's concept of evolution on the basis of physical lows and formulate the general rules of the evolution of complex systems, up to technics and social systems. On this path, we managed to get some new ideas about evolution.

a. In any natural system where there is a suitable building material, forces of attraction-repulsion can arise between dissimilar objects. Based on these forces, a potential well arises, in which oscillatory processes occur. If a pair of such objects dissipates the initial vibration energy to a minimum, then a new stable natural object appears in the depths of the potential well. This object retains in its internal structure the characteristic properties of the original objects, but at the same time displays new properties that allow it to effectively act both on the original world and on the surrounding worlds [2]. From the point of view of statistical mechanics, the emergence of a new complex object in the system means that evolution limits the spatial freedom of movement of the original objects. The translational degrees of freedom characteristic of the thermal motion of particles are transformed into oscillatory degrees of freedom, describing a higher degree of organization of the mechanical motion of objects. Thus, the ordering of the mechanical system takes place. According to Galimov, this is the main result of the evolutionary process [1].

b. Natural objects encounter by chance due to chaotic movement in a neutral environment, where they are immersed. A new evolutionary product will also be immersed there. It 
will certainly be destroyed by the general chaotic movement in the system. But from the decay products of a complex object, new similar objects can be formed without requiring an inexhaustible pool of initial building materials. Thus, chaotic movement brings death to complex natural objects. At the same time, such death is a necessary condition for the emergence and maintenance of life of the products of evolution [3].

c. For a randomly formed evolutionarily valuable natural object to be repeated in history, despite the destructive impact of world chaos, it is necessary to simultaneously emerge and preserve a separate auxiliary structure in the environment. Such a structure is obliged to capture and store the structural plan and history of the origin of this object. This important evolutionary mechanism has been called genetic memory [4]. The genetic code known from biochemistry is a private document of the genetic memory for all living things.

The following are observations illustrating the universal laws of evolutionary processes in systems of various levels of complexity. These observations are described in detail in the author's book [5].

\section{Prebiological World of Evolutionarily Promising Polymers}

Computer experiments were carried out on a model system where molecules of amino acids, nitrogenous bases, and ATP are immersed in a neutral medium [6]. Chaotic thermal motion occurs in the environment. The kinetic energies of particles are distributed approximately according to Boltzmann. This allows particles to collide accidentally. The forces of attraction-repulsion arising between them allow the particles to combine into more complex structures, dissipating the energy of chemical bonds outside. More energetic collisions destroy complex structures, returning the original building material to the system. It is considered that amino acids are independently combined into polypeptides, and polynucleotides are formed under the influence of polypeptides. A structural correspondence is imposed on the system: four amino acid residues are connected by intermolecular forces with the corresponding four nitrogenous bases. This constitutes a primitive hypothetical genetic code, simple and non-degenerate. The historical fate of emerging and disintegrating polymer chains is traced. This considers not only the strength of chemical bonds between amino acid residues in polypeptides, but also additional non-chemical bonds between the radicals of adjacent residues [7] Under these conditions, the system accumulates and retains a pool of complex structures that resemble modern protein structures in their complexity. The degree of complexity of the evolutionarily developing world was assessed by the richness of oscillatory degrees of freedom in the system [8].

It is noted that in such a primitive system, structures of polynucleotides are the carrier and translator of genetic memory.
As is known from modern data of biochemistry and forensic science, the structures of RNA and DNA are destroyed by heat movement much more slowly than the structures of proteins. This gives polynucleotides the ability to store the memory of the events of the random emergence of structures such as proteins that are successful for the future. Such structures in future worlds will play the most important role of enzymes, under the control of which biochemical processes in already living matter will go. This model world considers that new polynucleotides arise from molecules of nitrogenous bases and from fragments of destroyed nucleotides. This process is catalytically driven by long chains of polypeptides. Consequently, polypeptides also store elements of genetic memory in their structures. The model chemical system took care of storing its genetic memory not in one store, but in two. It is more reliable this way. This is how genetic memory is arranged in more complex worlds that have evolved evolutionarily. More on this is done below.

\section{Compartmentation was the most Important Stage in the Evolution of the Chemical World}

If the primitive model described above were adequate to the state of the early Ocean, then the chances of the emergence of living matter on our Planet would be exactly zero. Scattered amino acids or scraps of a degraded polypeptide must accidentally find a suitable polynucleotide store of memory of a successful polypeptide structure to reproduce that structure again. But a suitable strand of polynucleotide can float freely across the planet. And the act of reproducing the achievements of evolution will not take place. No, that does not work. At the final conference of the "Evolution of the Biosphere" Program, a hypothesis was proposed, in which the entire set of evolutionary rules outlined above is seen. The hypothesis looks like this.

A chance encounter of future biopolymers with products of destruction of geological structures was to take place. These products turned out to be weathering micropores in coastal rocks. The ocean tide flooded these micro pores with a solution of prebiological polymers, which were mixed with randomly formed lipids. A drop of water, enclosed in a lipid membrane, arose in a micro pore under the catalytic effect of polypeptides. In one of the next tides, an ocean wave could wash out a ready-made prototype of a living cell from the micropore. In this complex structure, under the protection of a shell, pure ocean water is stored, littered with polypeptides and polynucleotides, capable of both selfreproduction and the accidental creation of even more successful evolutionary structures. No need to look for random encounters all over the ocean. Everything you need is already in the household. And the primitive "cell" unconsciously seeks opportunities to complicate its inner world.

Let us pay attention to two circumstances in this hypothetical process. 
a. There is a chance meeting of two completely dissimilar natural objects. This is a portion of ocean water, where the evolutionary process of chemical creation is constantly taking place, and the product of the destruction of the structure of the mineral. The physical forces of attraction arise. Surface tension forces form a single drop of water, allowing that drop to randomly create with all its chemical objects, including lipids. Then the ocean tears off this drop from the cozy micro pore.

b. The structures of polynucleotides still serve as the carrier of genetic memory. However, now these structures have become more complex under the influence of polypeptides, which have accidentally learned to organize a lipid membrane around them. These functionally complicated polypeptides also store part of the cell's genetic memory. They know very well which of the nitrogenous bases and in what sequence should be combined with each other. Enzymes know even better which chemical compounds need to be beneficially influenced to accelerate biochemical processes. Now the cell, through a translucent shell, absorbs useful building material from the environment and throws useless products of dying of complex structures into the ocean. The cage is bursting open, it is ready to burst. But at the same time, clever precursors of enzymes organize not cell death, but the emergence of two new copies of the initial cell. An evolutionary leap is taking place. A drop of ocean water in a greasy shell begins to resemble something alive.

c. Comparing this picture with the process of increasing complexity in the primitive chemical world, we are convinced of the validity of the guess made in work [2]. The unconscious goal of evolution is further evolution, which involves the successful products of the previous stages of evolution. It seems that this is not a tautology, but a statement of an important fact: evolution is the most economical development process. Unlike the processes of degradation and revolution, it does not destroy anything to the ground, but uses the accumulation of complex structures and the products of their partial decay to create new, even more complex and effective structures.

\section{Symbiosis, Eukaryotes, Multicellular Organisms}

It would be strange if a physicist told readers of a biomedical journal about the details of the evolutionary origin of symbiosis, eukaryotes, and multicellular organisms. Better to focus on identifying the general rules of evolution in creating and maintaining the existence of these complex living systems. In symbiosis, two organisms independently store and implement their genetic mechanisms. They unite for the sake of mutual assistance if they find themselves in unfavorable environmental conditions. For example, lichen is a symbiosis of fungi and algae. In the harsh conditions of lack of moisture and light, lichen can live for a very long time as a single organism. However, when conditions improve, the lichen breaks down into the original algae and fungi. In this case, the genetic memory of the lichen does not disappear. When living conditions deteriorate, fungi and algae may accidentally meet again and remember that their organisms are well suited for joint survival. A complex natural object will appear again according to all the rules of evolution. This possibility is realized only because the genetic memory of the lichen is created and reliably stored in two different storages - in the structures of two different organisms.

The modern living cell is the result of the anchorage of an ancient symbiotic structure. It is assumed that once mitochondria, vacuoles and other cellular organelles existed as independent unicellular organisms. Having united symbiotically, they realized that for the sake of successful survival and reproduction, it was better to connect forever. And together they created not only the structure of a complex organism, but also a deeply divided genetic memory of the entire cell. This memory is stored not only in the nucleus, but also in individual organelles. All these elements of shared memory are included during cell division. The structures of numerous polynucleotides help the newly formed proteins to remember their structures, and enzymes remember the whole process of doubling the living material of the cell and guide this process. Multicellular organisms in terms of organizing their genetic memory are not much different from modern cells. The evolutionary leap was associated with the complication of the reproduction process, which is necessary for the successful survival of populations. There were cells of a special structure and purpose - sex cells. The storage of these cells with halves of genetic information was entrusted to individuals of two different sexes. Thus, the likelihood of damage to valuable information was sharply reduced.

It is well known that Nature does not at all care about the preservation of every living individual. But Nature values the results of each evolutionary process and takes care of reproducing the results obtained. For this, an evolutionary product that has arisen by chance is supplied with a genetic memory mechanism, where the entire history of a given branch of evolution is laid down together with the technological plan for the reproduction of the product. And just in case, out of caution, Nature places genetic memory not in the evolutionary product itself, but somewhere separately. Hence the impression is that Nature values evolution more than revolution, stagnation and degradation. Again, for reasons of economical handling of matter. The revolution destroys forever some of the previously accumulated structures. The stagnation of the system leads to the constant accumulation of harmful products outside. Degradation ultimately turns the entire system into a harmful entropic product. On the other hand, Nature cannot deal with the evolutionary ordering and complication of the entire Universe at the same time. This would lead to the complete elimination of chaotic forms of motion, that is, to the cessation of 
all evolutionary processes in general. Therefore, Nature willingly supports evolutionary processes only in individual material systems.

\section{Virus}

The work [9] is devoted to this branch of evolution. The hypothesis about the early origin of viruses, before the appearance of cellular and multicellular organisms, was considered. Based on the experience of modeling the evolution of biopolymers, it has been shown that in the primitive world of polypeptides and polynucleotides, a modern virion could hardly arise and expect the appearance of a cell suitable for its reproduction with a certain structure of its shell. Chaotic heat movement in the early Ocean would first destroy the protein capsid of the virion, and then its polypeptide center. Consequently, only the modern cell itself can create a virion based on its violent protein and nucleotide life. A cell creates such a virion either in defense of itself or to its death to help such objects multiply in future cells of the same structure. Who is to blame for this and how is it done today, when we are concerned about the emergence of new coronaviruses?

The fault is the modern degenerate genetic code, in which one codon can encode several amino acid residues. This sometimes disrupts the translation of structural information, which is usual for the cell. As a result of the mutation, a slightly altered protein text appears, and the protein chain is not separated from the DNA template, becoming a stable shell of the virion. It breaks out, accidentally falls into the parental type cell and begins to direct the process of self-reproduction there due to the materials of the living cell. As a result, the exhausted cell dies. We notice that in the evolutionary process of the emergence of a new virus, genetic memory is distributed among completely different objects. Part of the memory is stored by the virion, knowing what type of cell it can enter for reproduction. Part of the memory is stored by a cell of a certain type, knowing which virion it can help in its reproduction. A very reliable storage system for genetic memory. We bow before the wisdom of Nature.

\section{Technogenic Sphere}

The evolution of the biosphere has led to the emergence of a new natural object - the Homo sapiens population. This is a very serious evolutionary stage in the development of the Earth. Now, not only Nature unconsciously accidentally creates and not accidentally preserves objects valuable for further evolution. Now a person is more likely to consciously participate in this process, becoming precisely rational. Thus, he overcomes some of the inconveniences of his existence on the planet he accidentally got. Primitive man learned to get edible roots with a stick. He learned how to get meat from small animals using a sharpened stone. He learned how to tie brushwood for a fire with a vine. And still, the problem of family nutrition remained difficult to solve. He wanted to get a large animal, but it was dangerous to approach it with a stick. A technical problem is solved by invention. It is difficult to create a new tool with specified properties from scratch. However, there are evolutionary rules for obtaining an object with new useful qualities. It is necessary to use ready-made objects and successfully connect them to each other. The inventor tied a sharpened stone to a stick with a vine. This was the first ax. With such a tool it is no longer scary to approach a large beast. The beast will have a bad time. And the family will be better fed.

Then another important rule of evolution applies. It is necessary to form a genetic memory about the origin of the stone ax. Memory is not formed in the ax itself; it would be unreasonable and dangerous - the ax broke and the memory disappeared. Memory is formed in a completely different place - in the mind of the inventor. It is no longer necessary to carefully store the axes. It is enough to preserve the memory of the material and the manufacturing technique. And for this, the population has a completely different tool - oral speech for the transmission of traditions to future generations. In the future, this tool has vigorously evolved and gave rise to a completely new object - printing. The great Russian scientist V.I. Vernadsky considered the appearance of printing as the most important stage in the evolution of the noosphere. The author of this review is extremely pleased to work at the Vernadsky Institute and to consider this great thinker his heavenly patron.

\section{Social Systems}

The animal Homo sapiens, being a social animal, lives in social groups. These groups (families, tribes, countries, civilizations) are in motion, both in space and in historical time. Groups collide with each other because of chance encounters or mutual attraction. The nature of collisions can be very varied. This is the exchange of necessary goods, trade. This is an exchange of lexicons and grammars. Exchange of mentality. Forced removal of goods, robbery, war. War of mentalities. In the latter cases, collisions result in repulsion. We can say that between social groups there are forces of attraction-repulsion, albeit not of a physical nature. The energy of attraction-repulsion arises, not of a physical, but of a mental nature. Therefore, it can be expected that there is either a temporary increase or a temporary decrease in the energy of interaction, mental and emotional temperature. In the latter case, new types of societies may arise, with more complex structures than the structures of the original societies. These processes are analogous to evolutionary processes in mechanical and chemical systems.

The book [5] examines in detail the historical processes that evolutionarily led to the emergence of a unique civilization called differently: the Moscow principality, the Moscow kingdom, the Russian Empire, the Soviet Union. Now it is the Russian Federation. The uniqueness of this civilization lies in the fact that the ethnic 
groups included in it never fight each other. Not with the help of weapons, not with the help of mentality, religious beliefs, cultures. In no case can it be argued that in this Civilization there is peace and quiet and God's grace. For various reasons, external and internal, there are explosions of mental energies. It happens that an empire falls apart. And then in all the provinces that fell away from Russia, which until recently were successfully developing under the influence of the metropolis, wars begin. Wars are civil, as now in Ukraine, and there are also interethnic wars, as now in the South Caucasus.

It is important to note the manifestation of evolutionary patterns in these processes.

a. When two objects evolutionarily unite, a new object appears that can more effectively influence the surrounding systems. At the same time, evolution does not change the internal properties of the original objects too much. Therefore, when external or internal conditions change, a successful complex object disintegrates into initial ones, these initial objects lose a lot, become less capable. Ukraine can be recommended as an example.

b. During the evolutionary formation of an empire, genetic memory appears in the form of oral and written legends, historical annals, and sections of the humanities. The peak form of the empire's genetic memory is its great literature, which absorbed the epics of the ethnic groups that entered the empire. Literature is the most important component of the general culture of the empire. Therefore, after the collapse of the Russian Empire, the peoples again gravitated towards a common great culture and recreated the empire. Now Russia is restoring empire and civilization in its Federation.

Historical illustrations of these statements can be found in the book "Country: Ideology: Evolution" [5].

\section{ISSN: 2574-1241}

DOI: 10.26717/BJSTR.2020.32.005209

VA Dementiev. Biomed J Sci \& Tech Res

This work is licensed under Creative Commons Attribution 4.0 License

Submission Link: https://biomedres.us/submit-manuscript.php

\section{Conclusion}

Consideration of the patterns common to evolution in systems of any complexity level allows us to look with some optimism at the prospects for the future development of Mankind. Humanity constitutes a single system as part of the Earth's biosphere. Ecosystems develop and exist spontaneously in the conditions set by Nature. However, Humanity, being partially intelligent, is capable of directionally changing the internal conditions of its existence. This can lead both to the destruction of Humanity and to stagnation. But if Humanity realizes the possibilities and patterns of the evolutionary paths of its internal development, then a more prosperous and successful existence awaits it. The author of the review expressed this hope in the book [5] and gave detailed arguments in support of his correctness.

\section{References}

1. EM Galimov (2006) Phenomenon of Life. Origin and Principles of Evolution. URSS Publ. Hall, Moscow (in Russian). Engl. Translation: Geochemistry International 44(1): S1-S95.

2. VA Dementev (2014) The Driving Forces of Evolution. Geochemistry International 52(13): 1146-1189.

3. VA Dementiev (2020) How to: the origin of life or the rise of life and death? Biomedical Journal of Scientific \& Technical Research 26(1).

4. VA Dementiev (2020) The Formation of Genetic Memory at Various Stages of the Evolution of the Earth. Journal of Current Trends in Physics Research and Applications 1(1): 107.

5. VA Dementiev. Country: Ideology: Evolution.

6. VA Dementiev (2018) Origin of the simplest genetic code as an evolutionary stage of the Earth. Geochemistry International 56(1): 6570.

7. VA Dementiev (2018) Interaction of radicals in polypeptides. Current Research in Biopolymers: CRBP-101.

8. VA Dementiev (2018) Quantitative description of the course of chemical evolution. Current Research in Biopolymers: CRBP-108.

9. VA Dementiev (2020) Virus as evolutionary product of the world of biopolymers. Biomedical Journal of Scientific \& Technical Research 27(4).

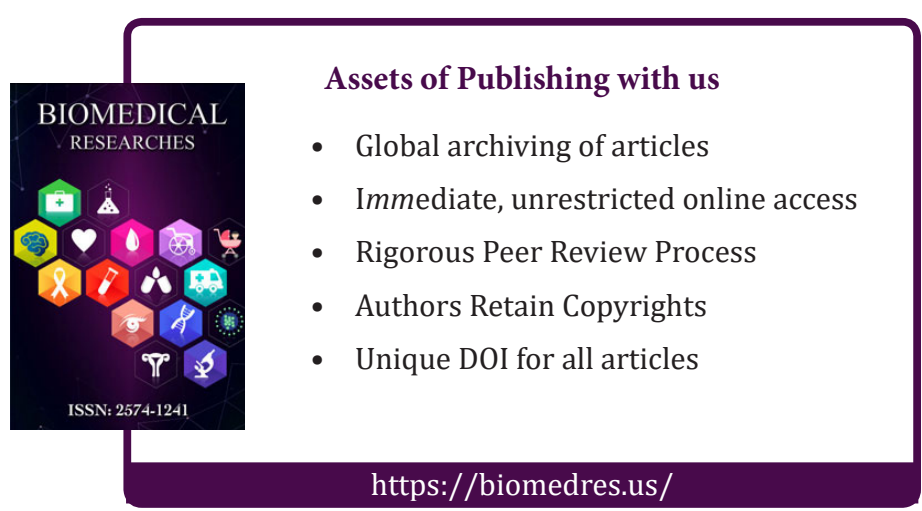

